\section{Bevacizumab Combined With Weekly Paclitaxel, Pegylated Liposomal Doxorubicin, or Topotecan in Platinum-Resistant Recurrent Ovarian Cancer: Analysis by Chemotherapy Cohort of the Randomized Phase III AURELIA Trial}

To THE EDITOR: In a previous issue of Journal of Clinical Oncology, we reported results from the open-label, randomized phase III AURELIA (Avastin Use in Platinum-Resistant Epithelial Ovarian Cancer) trial demonstrating that combining bevacizumab with singleagent chemotherapy for treatment of platinum-resistant recurrent ovarian cancer (PROC) significantly improved progression-free survival (PFS), the primary end point, as well as the objective response rate (ORR) and the patient-reported outcome end point of abdominal/GI symptoms in the intent-to-treat population of 361 patients. ${ }^{1,2}$ We observed no significant difference in overall survival (OS) between treatment arms, though the trial was not designed for us to formally compare OS. In addition, the extensive cross-over of $40 \%$ of patients to bevacizumab from chemotherapy alone complicated interpretation.

In AURELIA, investigators chose their preferred chemotherapy (from weekly paclitaxel, pegylated liposomal doxorubicin [PLD], or topotecan) for each patient before randomization. Enrollment onto each cohort was capped to enable meaningful evaluation of each regimen. Patients were stratified by selected chemotherapy but not randomly assigned among chemotherapy regimens. In all three chemotherapy cohorts, PFS was significantly improved by adding bevacizumab to chemotherapy, consistent with the overall results. PFS hazard ratios (HRs) were 0.46 (95\% confidence interval [95\% CI], 0.30 to 0.71 ) in the paclitaxel cohort (median, $10.4 v 3.9$ months; Fig 1A), 0.57 (95\% CI, 0.39 to 0.83 ) for PLD (median $5.4 v 3.5$ months favoring bevacizumab-containing therapy), and 0.32 ( $95 \% \mathrm{CI}, 0.21$ to 0.49 ) for topotecan (median $5.8 \times 2.1$ months, respectively).

ORR by RECIST was higher with bevacizumab-containing therapy versus chemotherapy alone in the paclitaxel cohort $(53.3 \% \mathrm{v}$ $30.2 \%$, respectively; difference, $23.1 \%$; $95 \%$ CI, $1.7 \%$ to $44.5 \%$ ) and the topotecan cohort (17.0\% $v 0.0 \%$; difference, $17.0 \%$; $95 \%$ CI, $5.1 \%$ to $28.9 \%)$, with a less pronounced effect in the PLD cohort $(13.7 \% \mathrm{v}$ $7.8 \%$; difference, $5.9 \%$; $95 \% \mathrm{CI},-7.2 \%$ to $19.0 \%$ ).

Analyses of patient-reported outcomes were based on a responder analysis approach to compare the proportion of patients in each treatment arm achieving $\geq 15 \%$ improvement at week 8 or 9 in an abdominal/GI symptom subscale of the European Organisation for Research and Treatment of Cancer Quality of Life Questionnaire OV28. In each cohort, numerically more patients receiving bevacizumab-containing therapy than chemotherapy alone achieved $\geq 15 \%$ improvement in abdominal/GI symptoms. The proportions were $25.0 \%$ versus $13.0 \%$, respectively, in the paclitaxel cohort (difference, $12.0 \%$; $95 \%$ CI, $-4.9 \%$ to $28.9 \%$ ), $20.0 \%$ versus $8.8 \%$ in the topotecan cohort (difference, $11.2 \%$; $95 \% \mathrm{CI},-3.2 \%$ to $25.7 \%$ ) and $21.1 \%$ versus $6.8 \%$ in the PLD cohort (difference, $14.3 \%$; $95 \%$ CI, $0.9 \%$ to $27.6 \%$ ).

We found no significant difference in OS between treatment arms in any of the chemotherapy cohorts; this was consistent with the overall population. Unadjusted HRs were 0.91 (95\% CI, 0.62 to 1.36) for PLD (median, 13.7 months with bevacizumab-containing therapy

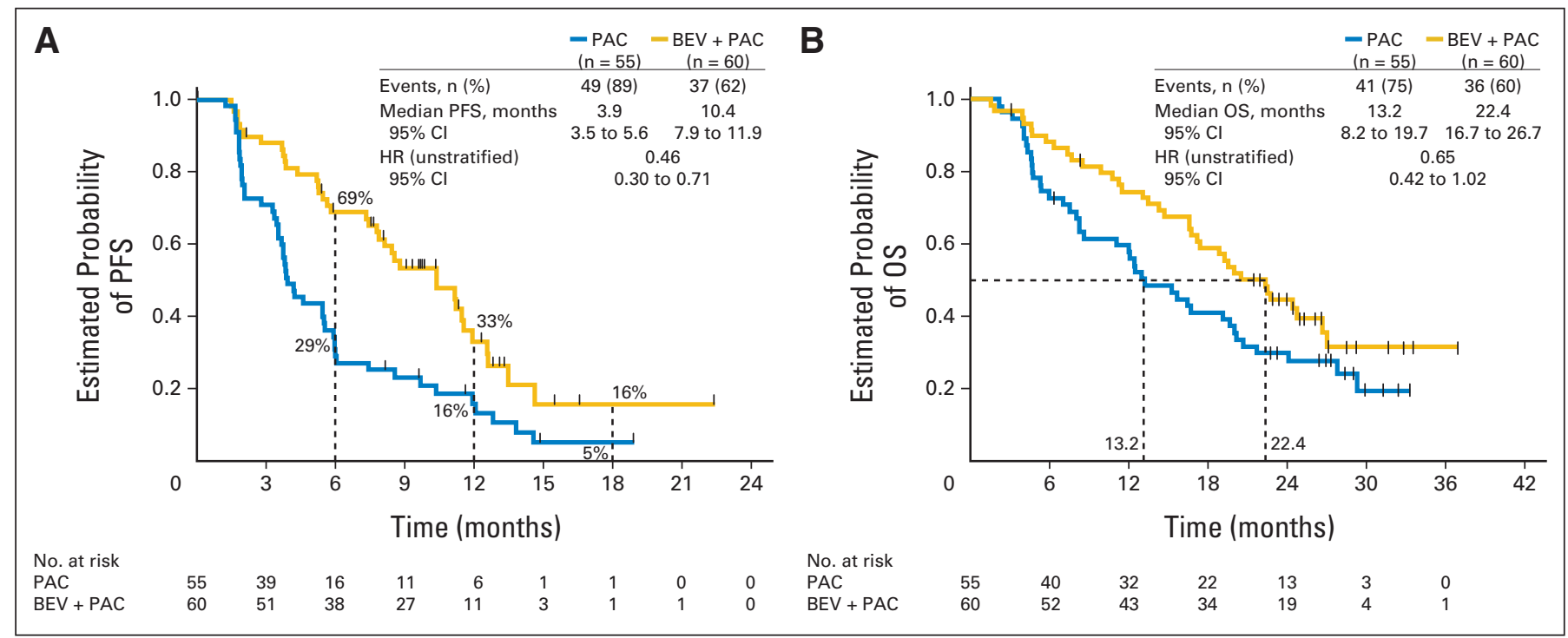

Fig 1. Weekly paclitaxel (PAC) cohort. (A) Progression-free survival (PFS) with a data cutoff date of November 14, 2011. (B) Overall survival (OS) with a data cutoff date of January 25, 2013. BEV, bevacizumab; HR, hazard ratio. 


\begin{tabular}{|c|c|c|c|c|c|c|}
\hline \multirow[b]{2}{*}{ Characteristic } & \multicolumn{2}{|c|}{ PLD } & \multicolumn{2}{|c|}{ Topotecan } & \multicolumn{2}{|c|}{ Weekly Paclitaxel } \\
\hline & $\begin{array}{l}\text { No Bevacizumab } \\
\quad(\mathrm{n}=64)\end{array}$ & $\begin{array}{c}\text { Bevacizumab } \\
(\mathrm{n}=62)\end{array}$ & $\begin{array}{c}\text { No Bevacizumab } \\
(\mathrm{n}=63)\end{array}$ & $\begin{array}{l}\text { Bevacizumab } \\
(n=57)\end{array}$ & $\begin{array}{c}\text { No Bevacizumab } \\
(\mathrm{n}=55)\end{array}$ & $\begin{array}{c}\text { Bevacizumab } \\
\quad(n=60)\end{array}$ \\
\hline Recruitment & \multicolumn{2}{|c|}{ October 2009-October 2010} & \multicolumn{2}{|c|}{ October 2009-April 2011} & \multicolumn{2}{|c|}{ October 2009-April 2011} \\
\hline \multicolumn{7}{|l|}{ Age, years } \\
\hline Median (range) & $62(32-77)$ & $63.5(39-78)$ & $61(35-84)$ & $60(26-80)$ & $60(25-80)$ & $60(25-79)$ \\
\hline \multicolumn{7}{|l|}{ Histology at diagnosis* } \\
\hline Serous or adenocarcinoma & $49(77)$ & $53(85)$ & $55(87)$ & $50(88)$ & $48(87)$ & $53(88)$ \\
\hline Clear cell & $6(9)$ & $1(2)$ & $3(5)$ & $1(2)$ & $3(5)$ & $2(3)$ \\
\hline FIGO stage III or IV & $52(81)$ & $56(90)$ & $56(89)$ & $55(96)$ & $48(87)$ & $54(90)$ \\
\hline \multicolumn{7}{|l|}{ Histologic grade at diagnosis } \\
\hline 1 & $4(6)$ & $1(2)$ & 2 (3) & $2(4)$ & $3(5)$ & $7(12)$ \\
\hline 2 & $14(22)$ & $15(24)$ & $17(27)$ & $20(35)$ & $17(31)$ & $18(30)$ \\
\hline 3 & $40(63)$ & $36(58)$ & $40(63)$ & $27(47)$ & $25(45)$ & $31(52)$ \\
\hline Missing & $6(9)$ & $10(16)$ & $4(6)$ & $8(14)$ & $10(18)$ & $4(7)$ \\
\hline Two previous chemotherapy regimens & $21(33)$ & $16(26)$ & $29(46)$ & $23(40)$ & $28(51)$ & $33(55)$ \\
\hline \multicolumn{7}{|l|}{ Prognostic factors* } \\
\hline \multicolumn{7}{|l|}{ Platinum-free interval, months } \\
\hline$<3$ & $14(22)$ & $18(29)$ & $16(25)$ & $17(30)$ & $16(29)$ & $16(27)$ \\
\hline$\geq 3$ & $49(77)$ & $44(71)$ & $47(75)$ & $39(68)$ & $38(69)$ & $44(73)$ \\
\hline \multicolumn{7}{|l|}{ ECOG performance status } \\
\hline 0 & $38(59)$ & $34(55)$ & $34(54)$ & $35(61)$ & $27(49)$ & $38(63)$ \\
\hline 1 or 2 & $26(41)$ & $28(45)$ & $28(44)$ & 22 (39) & $26(47)$ & $20(33)$ \\
\hline Baseline CA-125 $\geq 100 \mathrm{U} / \mathrm{mL}$ & $46(72)$ & $45(73)$ & $46(73)$ & $46(81)$ & $42(76)$ & $45(75)$ \\
\hline Ascites at baseline & $20(31)$ & $24(39)$ & $19(30)$ & $20(35)$ & $15(27)$ & $15(25)$ \\
\hline \multicolumn{7}{|l|}{ Measurable disease, SLD, cm } \\
\hline$<1$ or no lesion & $13(20)$ & $11(18)$ & $13(21)$ & $11(19)$ & $12(22)$ & $15(25)$ \\
\hline 1 to $<5$ & $22(34)$ & $23(37)$ & $25(40)$ & $25(44)$ & $13(24)$ & $17(28)$ \\
\hline$\geq 5$ & $29(45)$ & $28(45)$ & $25(40)$ & $21(37)$ & $30(55)$ & $28(47)$ \\
\hline
\end{tabular}

$v 14.1$ months with chemotherapy alone) and 1.09 (95\% CI, 0.72 to 1.67) for topotecan (median, $13.8 v 13.3$ months). However, a more pronounced treatment effect on OS was seen in the paclitaxel cohort (unadjusted HR, 0.65; 95\% CI, 0.42 to 1.02 ; median $22.4 v 13.2$ months; Fig 1B). The extent of cross-over to bevacizumab from chemotherapy alone was similar in the three chemotherapy cohorts (paclitaxel, 38\%; PLD, 39\%; topotecan, 41\%).

These findings, albeit generated in exploratory analyses of small subgroups, generate two questions. First, are there differences in the activity of the three chemotherapy regimens in PROC? And, second, is there an optimal chemotherapy partner for bevacizumab in PROC?

In the chemotherapy-only arm, numbers of PFS events, median PFS, and ORR seemed to differ between cohorts. Topotecan, typically given weekly, seemed less active than weekly paclitaxel, with intermediate results for PLD. This observation is aligned with data suggesting suboptimal efficacy of weekly topotecan, ${ }^{3}$, whereas weekly paclitaxel induces high ORRs but disappointing PFS. ${ }^{4-6}$ However, median OS with chemotherapy alone was similar between cohorts and consistent with historical data.

A major limitation of such comparisons is the lack of randomization to chemotherapy cohorts. One may expect imbalances in measured and unmeasured potentially prognostic factors among cohorts given that clinical, disease, and patient characteristics presumably influenced chemotherapy selection. In general, however, baseline characteristics were balanced, except for the proportion of patients who had received two previous chemotherapy regimens (Table 1). To further explore prognostic factors for OS, we fitted a multivariable Cox model starting with all covariates significant at $15 \%$ in a univariable model. Backwards selection at the $5 \%$ level provided the final model. Chemotherapy partner, performance status, ascites, disease measurability, baseline cancer antigen-125 value, and platinum-free interval were significant prognostic factors for OS. These factors were evenly distributed among chemotherapy cohorts and between treatment arms; this result suggested that the striking results with weekly paclitaxel were unlikely to be explained by patient population imbalances. Furthermore, OS in the intent-to-treat population after we adjusted for these prognostic factors was consistent with results of the unadjusted primary analysis.

In each chemotherapy cohort, the bevacizumab-chemotherapy combination significantly improved PFS, the primary end point, compared with chemotherapy alone, and it should be considered a new standard option for PROC. The US Food and Drug Administration and the European Commission approved bevacizumab combined with chemotherapy for PROC on the basis of results from AURELIA. However, interpretation of OS is less straightforward, complicated by study-design features, such as investigator-selected chemotherapy and optional crossover to bevacizumab; other postprogression therapy; and the lessconsistent treatment effects between cohorts. We observed no difference 
in OS between treatment arms in the PLD and topotecan cohorts, but Kaplan-Meier curves for OS were clearly separated in the paclitaxel cohort. Experience with metastatic breast cancer suggests that bevacizumab combined with weekly paclitaxel may be more active than other backbones for chemotherapy. ${ }^{7}$ Combining these two agents may enhance their antiangiogenic effects and potentially account for observations in AURELIA. In the front-line setting, this hypothesis was not supported in exploratory analyses of the GOG-262 trial. ${ }^{8}$ However, the role of frontline weekly paclitaxel remains controversial. ${ }^{9,10}$

The main limitation of these exploratory analyses is that assessing individual chemotherapy partners for bevacizumab was not an objective of AURELIA. However, exploring consistency of effect in clinical trials is important, not necessarily for guiding treatment practice, but at least for hypothesis generation, particularly if a plausible biologic explanation for differences exists. Although consistency, on the basis of treatment effect estimates and 95\% CIs, was seen between cohorts, the effect on PFS, ORR, and OS of combining bevacizumab with weekly paclitaxel was remarkable. These hypothesis-generating observations should be considered when investigators design new trials in PROC.

\section{Andres M. Poveda}

Grupo Español de Investigación en Cáncer de Ovario (GEICO) and Instituto Valenciano de Oncologia, Valencia, Spain

\section{Frédéric Selle}

Groupe d'Investigateurs Nationaux pour l'Etude des Cancers Ovariens (GINECO), University Pierre et Marie Curie-Sorbonne, Tenon Hospital, Hôpitaux Universitaires de l'Est Parisien-Assistance Publique-Hôpitaux de Paris, and Alliance Pour la Recherche en Cancérologie, Paris, France

\section{Felix Hilpert}

Arbeitsgemeinschaft Gynaekologische Onkologie (AGO) and Klinik für Gynäkologie und Geburtshilfe, Kiel, Germany

\section{Alexander Reuss}

AGO and Coordinating Center for Clinical Trials, Marburg, Germany

\section{Antonella Savarese}

Multicenter Italian Trials in Ovarian Cancer and Gynecologic Malignancies and Regina Elena National Cancer Institute, Rome, Italy

\section{Ignace Vergote}

Belgian Gynaecological Oncology Group and University Hospital Leuven, Leuven, Belgium

\section{Petronella Witteveen}

Dutch Gynaecological Oncology Group and University Medical Center Utrecht, Utrecht, the Netherlands

\section{Aristotelis Bamias}

Hellenic Cooperative Oncology Group and University of Athens, Athens, Greece

\section{Nana Scotto and Lada Mitchell}

F. Hoffmann-La Roche, Basel, Switzerland

\section{Eric Pujade-Lauraine}

GINECO and Université Paris Descartes, Assistance Publique-Hôpitaux de Paris, Paris, France

\section{ACKNOWLEDGMENT}

The trial and medical writing were supported by F. Hoffmann-La Roche. Written on behalf of the Groupe d'Investigateurs Nationaux pour l'Etude des Cancers Ovarien (GINECO) and the European Network of Gynaecological Oncological Trial Groups (ENGOT) — Gynecologic Cancer Intergroup (GCIG) investigators.

\section{AUTHORS' DISCLOSURES OF POTENTIAL CONFLICTS OF INTEREST}

Disclosures provided by the authors are available with this article at www.jco.org.

\section{REFERENCES}

1. Pujade-Lauraine $E$, Hilpert F, Weber B, et al: Bevacizumab combined with chemotherapy for platinum-resistant recurrent ovarian cancer: The AURELIA open-label randomized phase III trial. J Clin Oncol 32:1302-1308, 2014

2. Stockler MR, Hilpert F, Friedlander $M$, et al: Patient-reported outcome results from the open-label phase III AURELIA trial evaluating bevacizumabcontaining therapy for platinum-resistant ovarian cancer. J Clin Oncol 43:13091316, 2014

3. Sehouli J, Stengel D, Harter $P$, et al: Topotecan weekly versus conventional 5-day schedule in patients with platinum-resistant ovarian cancer: A randomized multicenter phase II trial of the North-Eastern German Society of Gynecological Oncology Ovarian Cancer Study Group. J Clin Oncol 29:242-248, 2011

4. Lortholary A, Largillier R, Weber B, et al: Weekly paclitaxel as a single agent or in combination with carboplatin or weekly topotecan in patients with resistant ovarian cancer: The CARTAXHY randomized phase II trial from Groupe d'Investigateurs Nationaux pour l'Etude des Cancers Ovariens (GINECO). Ann Oncol 23:346-352, 2012

5. Karlan BY, Oza AM, Richardson GE, et al: Randomized, double-blind, placebo-controlled phase II study of AMG 386 combined with weekly paclitaxel in patients with recurrent ovarian cancer. J Clin Oncol 30:362-371, 2012

6. Monk BJ, Poveda A, Vergote I, et al: Anti-angiopoietin therapy with trebananib for recurrent ovarian cancer (TRINOVA-1): A randomised, multicentre, double-blind, placebo-controlled phase 3 trial. Lancet Oncol 15:799-808, 2014

7. Miller K, Wang M, Gralow J, et al: Paclitaxel plus bevacizumab versus paclitaxel alone for metastatic breast cancer. N Engl J Med 357:2666-2676, 2007

8. Chan J, Brady M, Penson R, et al: Phase III trial of every-3-weeks paclitaxel vs. dose dense weekly paclitaxel with carboplatin +/- bevacizumab in epithelial ovarian, peritoneal, fallopian tube cancer: GOG 262 (NCT01167712). Int J Gynecol Cancer 23:9, 2013 (suppl 1; abstr 8)

9. Katsumata N, Yasuda M, Isonishi S, et al: Long-term results of dose-dense paclitaxel and carboplatin versus conventional paclitaxel and carboplatin for treatment of advanced epithelial ovarian, fallopian tube, or primary peritoneal cancer (JGOG 3016): A randomised, controlled, open-label trial. Lancet Oncol 14:1020-1026, 2013

10. Pignata S, Scambia G, Katsaros D, et al: Carboplatin plus paclitaxel once a week versus every 3 weeks in patients with advanced ovarian cancer (MITO-7): A randomised, multicentre, open-label, phase 3 trial. Lancet Oncol 15:396-405, 2014

DOI: 10.1200/JCO.2015.63.1408; published online ahead of print at www.jco.org on August 17, 2015 


\section{AUTHORS' DISCLOSURES OF POTENTIAL CONFLICTS OF INTEREST}

Bevacizumab Combined With Weekly Paclitaxel, Pegylated Liposomal Doxorubicin, or Topotecan in Platinum-Resistant Recurrent Ovarian Cancer: Analysis by Chemotherapy Cohort of the Randomized Phase III AURELIA Trial

The following represents disclosure information provided by authors of this manuscript. All relationships are considered compensated. Relationships are self-held unless noted. I = Immediate Family Member, Inst = My Institution. Relationships may not relate to the subject matter of this manuscript. For more information about ASCO's conflict of interest policy, please refer to www.asco.org/rwc or jco.ascopubs.org/site/ifc.

\section{Andres M. Poveda}

Consulting or Advisory Role: Roche, AstraZeneca, PharmaMar

Frédéric Selle

Consulting or Advisory Role: Roche, PharmaMar, MSD, AstraZeneca

Felix Hilpert

Honoraria: Roche, AstraZeneca, Janssen-Cilag, MSD, Teva

Neuroscience, Array BioPharma

Consulting or Advisory Role: Roche, AstraZeneca, Janssen-Cilag, MSD

Travel, Accommodations, Expenses: Roche, AstraZeneca

Alexander Reuss

Travel, Accommodations, Expenses: Roche, Boehringer Ingelheim

Antonella Savarese

No relationship to disclose

Ignace B Vergote

Research Funding: Roche

\section{Petronella Witteveen}

No relationship to disclose

Aristotelis Bamias

Honoraria: Roche

Consulting or Advisory Role: Roche

Research Funding: Amgen

\section{Nana Scotto}

Employment: F. Hoffmann-La Roche

Stock or Other Ownership: F. Hoffmann-La Roche

Travel, Accommodations, Expenses: F. Hoffmann-La Roche

Lada Mitchell

Employment: Roche

Stock or Other Ownership: Roche

Eric Pujade-Lauraine

Honoraria: Roche, AstraZeneca 